\title{
AhlX, an $N$-acylhomoserine Lactonase with Unique Properties
}

\author{
Pengfu Liu ${ }^{1,2}$, Yan Chen ${ }^{1}$, Zongze Shao ${ }^{3}$, Jianwei Chen ${ }^{1}$, Jiequn $\mathrm{Wu}^{1}$, Qian Guo ${ }^{1}$, Jiping Shi ${ }^{2}$, \\ Hong Wang $1, * \mathbb{D}$ and Xiaohe $\mathrm{Chu}{ }^{1, *}$ \\ 1 Collaborative Innovation Center of Yangtze River DeltaRegion Green Pharmaceuticals, \\ Zhejiang University of Technology, Hangzhou, Zhejiang 310014, China \\ 2 Shanghai Advanced Research Institute, Chinese Academy of Sciences, Pudong, Shanghai 201210, China \\ 3 Key Laboratory of Marine Biogenetic Resources, The Third Institute of Oceanography, \\ Ministry of Natural Resources, Xiamen 361005, China \\ * Correspondence: hongw@zjut.edu.cn (H.W.); chuxhe@zjut.edu.cn (X.C.); Tel.: +86-571- 8832-0622 (H.W.); \\ $+86-137-0682-8992$ (X.C.)
}

Received: 23 May 2019; Accepted: 25 June 2019; Published: 28 June 2019

\begin{abstract}
N$-Acylhomoserine lactonase degrades the lactone ring of $N$-acylhomoserine lactones (AHLs) and has been widely suggested as a promising candidate for use in bacterial disease control. While a number of AHL lactonases have been characterized, none of them has been developed as a commercially available enzymatic product for in vitro AHL quenching due to their low stability. In this study, a highly stable AHL lactonase (AhlX) was identified and isolated from the marine bacterium Salinicola salaria MCCC1A01339. AhlX is encoded by a 768-bp gene and has a predicted molecular mass of $29 \mathrm{kDa}$. The enzyme retained approximately $97 \%$ activity after incubating at $25^{\circ} \mathrm{C}$ for 12 days and $\sim 100 \%$ activity after incubating at $60^{\circ} \mathrm{C}$ for $2 \mathrm{~h}$. Furthermore, AhlX exhibited a high salt tolerance, retaining approximately $60 \%$ of its activity observed in the presence of $25 \% \mathrm{NaCl}$. In addition, an AhlX powder made by an industrial spray-drying process attenuated Erwinia carotovora infection. These results suggest that AhlX has great potential for use as an in vitro preventive and therapeutic agent for bacterial diseases.
\end{abstract}

Keywords: quorum sensing; quorum-quenching; $N$-acylhomoserine lactonase; marine; thermostable; salt tolerance

\section{Introduction}

Bacteria can monitor and respond to their population density using small secreted signaling molecules. The concentration of these molecules increases as the bacteria proliferate. Once it reaches a threshold, the accumulated signal molecules elicit the expression of specialized genes involved in bioluminescence [1], Ti plasmid transfer [2], antibiotic production [3], biofilm formation [4] or pathogenic process [5]. This cell population-dependent behavior is widely known as "quorum sensing (QS)" [6]. The most important QS mechanism identified to date relies on the signaling molecules $N$-acylhomoserine lactones (AHLs), all of which possess the same lactone ring but have different lengths and types of acyl-chains [7]. Most Gram-negative bacteria, including several pathogens such as Erwinia sp., Vibrio sp., Yersinia sp., Agrobacterium sp., and Pseudomonas sp., use AHLs to modulate their QS behaviors [7].

AHL lactonase catalyzes the hydrolysis of the lactone bond of AHLs and is able to quench AHL-dependent QS signaling. While several bacterial pathogens use QS system to regulate their virulence [2,8], the QS quenching activity of AHL lactonase has always been considered to be an important tool for bacterial disease control. The first AHL lactonase, AiiA, was identified from 
Bacillus sp. 240B1 in 2000 and is comprised of 250 amino acids. AiiA contains a typical "HXHXDH" metallohydrolase family motif and requires zinc ions for its activity. The heterologous expression of AiiA in Erwinia carotovora strain SCG1 inhibits the release of its AHL molecules and clearly attenuates its QS-dependent pathogenic processes towards Chinese cabbage, carrots, cauliflower, celery, potatoes, eggplant and tobacco [9]. The transgenic expression of AiiA in plants has been shown to significantly increase the resistance of the plants towards pathogens [10].

At present, apart from the aforementioned organisms, AHL lactonases have also been identified and isolated from Solibacillus sp., Arthrobacter sp., Klebsiella sp., Rhizobium sp., Ochrobactrum sp., Microbacterium sp., Chryseobacterium sp., Rhodococcus sp., Oceanicaulis sp., Pseudoalteromonas sp., Sulfolobus sp., Ruegeria sp., soil metagenomes and human tissues [11-13]. However, none of these enzymes have been developed as a commercially available AHL lactonase product for AHL quenching due to their low stability, impeding their further application as in vitro preventive and therapeutic agents for bacterial diseases.

In this study, an AHL lactonase from a marine bacterium, S. salaries MCCC1A01339, was identified and characterized. AhlX displays unique properties, including high temperature stability and high salt tolerance. AhlX can be also used to attenuate E. carotovora infection. To the best of our knowledge, AhlX is one of the most stable AHL lactonases identified to date and should have excellent potential for further biotechnological use.

\section{Results}

\subsection{Cloning of the Lactonase-Encoding Gene AhlX}

We previously identified a marine bacterium showing clear AHL-degrading activity as indicated in an A. tumefaciens NT1 bioassay [14]. This strain was isolated from the Indian Ocean and was initially identified and named as Halomonas salaria MCCC1A01339. However, since Halomonas salaria has been classified into the Salinicola genus and is named as Salinicola salarius now [15], we now refer to this strain as S. salarius MCCC1A01339. The strain generally requires $\geq 3 \% \mathrm{NaCl}$ for a good growth in lab. The reported phylogenetically closest strains to S. salaries MCCC1A01339 are S. salarius strain M27, as shown by $16 \mathrm{~S}$ rDNA alignment (>99\% identity), and S. salarius DSM 18044, as shown by $23 \mathrm{~S}$ rDNA alignment ( $>99 \%$ identity) (data not shown).

To clone the gene encoding the AHL-degrading enzyme from S. salarius MCCC1A01339, we obtained its draft genome sequence via next generation sequencing and subsequent assembly and annotation. From the assembled genome, 3853 genes were identified with an average length of $936 \mathrm{bp}$. The mean GC content in the gene region is $63.50 \%$. Subsequently, 2688 genes were further annotated. Analysis of the $S$. salaries MCCC1A01339 draft genome enabled us to identify a 768-bp gene (ahlX) encoding a potential AHL-degrading enzyme, with the encoded protein sharing the highest identity of 67\% to AttM from A. tumefaciens [16] and 31\% to AiiA from Bacillus sp. 240 B1 [9]. AhlX consists of 261 amino acids and has a predicted molecular mass of $29.2 \mathrm{kDa}$.

\subsection{Bioinformatic Analysis of AhlX}

As mentioned above, AhlX shares relatively low identity with AttM from A. tumefaciens [16] and AiiA from Bacillus sp. 240 B1 [9]. However, AhlX shares high identity with several predicted AHL lactonases in the database. For example, AhlX shares $92 \%$ identity with a predicted AHL lactonase (Gba-pALase) from a bacterium (Gammaproteobacteria strain MFB021) isolated from petroleum-contaminated soil [17]; 90\% identity with a predicted AHL lactonase (Sso-pAlase) from a bacterium (Salinicola socius) isolated from a salt mine (Perm region of Russia) [18]; and 83\% identity with a predicted AHL lactonase (Kav-pALase) from a bacterium (Kushneria avicenniae) isolated from a salty leaf [19].

We further determined the phylogenetic relationship between these AHL lactonases and other well-characterized AHL lactonases. AhlX and the randomly selected predicted AHL lactonases Gba-pALase, Sso-pAlase and Kav-pALaseare were observed to cluster into a small group that is 
phylogenetically distant from the well-characterized AiiA group from Bacillus sp. [9] and AidC from Chryseobacterium sp. StRB126 [20]. The phylogenetically closest and well-characterized AHL lactonases to AhlX were AhlK from Klebsiella pneumoniae [21] and AttM from A. tumefaciens [16] (Figure 1).

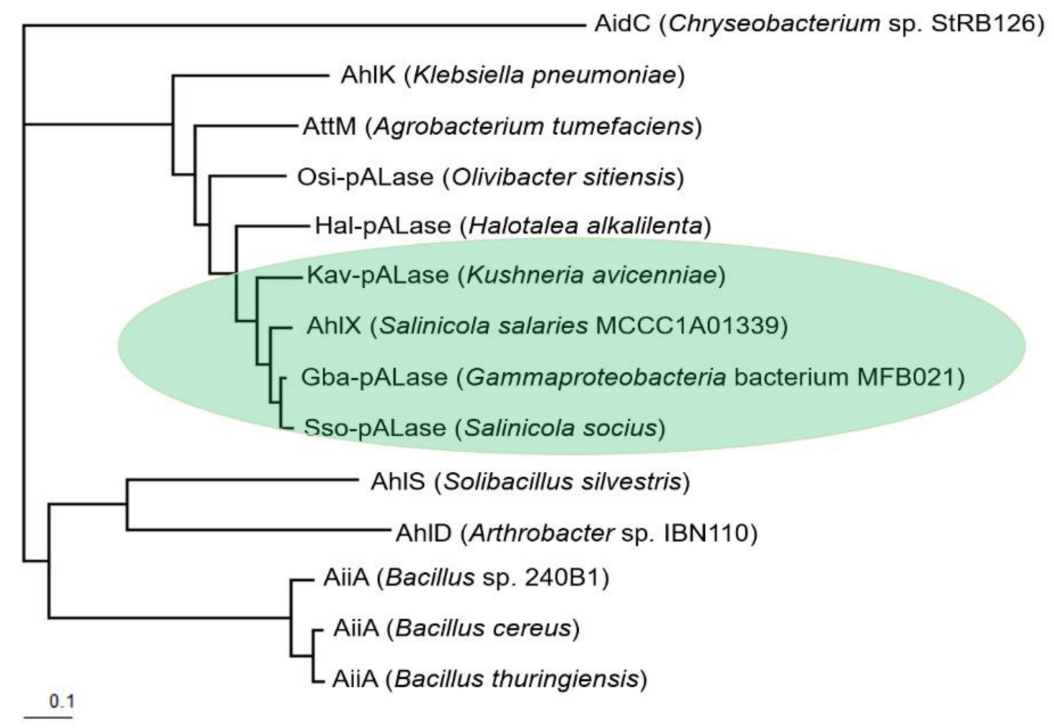

Figure 1. Phylogenetic tree of selected N-acylhomoserine lactone (AHL) lactonases. The AHL lactonase sequences used were AidC (BAM28988.1) from Chryseobacterium sp. StRB126; AhlK (AAO47340.1) from K. pneumoniae; AttM (AAL13075.1) from A. tumefaciens; Osi-pALase (predicted AHL lactonase; WP_028296339.1) from Olivibacter sitiensis; Hal-pALase (predicted AHL lactonase; WP_064123850.1) from Halotalea alkalilenta; Kav-pALase (SFC91482.1) from Kushneria avicenniae; AhlX from S. salaries MCCC1A10339; Gba-pALase (WP_035471581.1) from Gammaproteobacteria bacterium MFB021; Sso-pALase (WP_075571152.1) from Salinicola socius; AhlS (BAK54003.1) from Solibacillus silvestris; AhID (AAP57766.1) from Arthrobacter sp. IBN110; AiiA (AAF62398.1) from Bacillus sp. 240B1;AiiA (AAL98724.1) from Bacillus cereus and AiiA (AAL98718.1) from Bacillus thuringiensis. The AHL lactonases from halophilic bacteria are highlighted in light blue.

The multiple sequence alignment showed that, similar to other well-characterized AHL lactonases, AhlX, Gba-pALase, Sso-pAlase and Kav-pALase all contain the typical zinc binding motif " $\mathrm{H}^{102} \mathrm{XH}^{104} \mathrm{XD}^{106} \mathrm{H}^{107} \sim \mathrm{H}^{178}$ ", which is conserved in the metallo- $\beta$-lactamase superfamily [22,23]. Moreover, three additional residues $\left(\mathrm{D}^{200}, \mathrm{Y}^{203}\right.$ and $\left.\mathrm{H}^{245}\right)$, which have been shown to be crucial for the activity of AHL lactonases by interacting with the ligand or through substrate binding, were also observed in AhlX, Gba-pALase, Sso-pAlase and Kav-pALase. Notably, AhlX, Gba-pALase, Sso-pAlase and Kav-pALase harbor five conserved cysteines in their $N$-terminal region, including a $\mathrm{C}^{16} \mathrm{XXC}{ }^{19}$ motif (Figure 2).

While the 3D-structure of several AHL lactonases have been solved, we sought to elucidate the AhlX structure by template based mathematical modeling using the online protein structure and function prediction server I-TASSER (http://zhanglab.ccmb.med.umich.edu/I-TASSER) [24]. As shown in Figure 3a, AhlX contains a $\alpha \beta / \beta \alpha$ sandwich fold, with the helices located in the outer solvent-exposed layer and the $\beta$ sheets condensed in the core. This structure typically exists in various metallo- $\beta$-lactamase family proteins [25]. AhlX harbors 12 strands ( $\beta 1$, residues $5-18$; $\beta 2$, residues $28-41 ; \beta 3$, residues $44-49 ; \beta 4$, residues $72-74 ; \beta 5$, residues $96-100 ; \beta 6$, residues $118-121 ; \beta 7$, residues $154-156$; $\beta 8$, residues $162-166$; $\beta$, residues $168-175 ; \beta 10$ residues $182-188 ; \beta 11$ residues 194-198; and $\beta 12$ residues $240-244)$ and 10 helices $(\alpha 1$, residues 19-21; $\alpha 2$, residues $65-70 ; \alpha 3$, residues $71-88 ; \alpha 4$, residues $110-111 ; \alpha 5$, residues $123-130 ; \alpha 6$, residues $135-136 ; \alpha 7$, residues 146-147; $\alpha 8$, residues 205-210; $\alpha$, residues 220-236; and $\alpha 10$, residues $247-252)$. All of the residues $\left(\mathrm{H}^{102}, \mathrm{H}^{104}\right.$, $\mathrm{D}^{106}, \mathrm{H}^{107}, \mathrm{H}^{178}, \mathrm{D}^{200}, \mathrm{Y}^{203}$ and $\mathrm{H}^{245}$ ) that have been showed to be important in the activity of AHL 
lactonases are also illustrated and appear to be located on the flexible loop and buried inside the $\beta$ sheet core, forming an excellent active center (Figure $3 b, c)$. The $C^{16} X X C^{19}$ motif is observed to be located close to the active center, like a "door keeper" of the active center (Figure $3 b$ ).
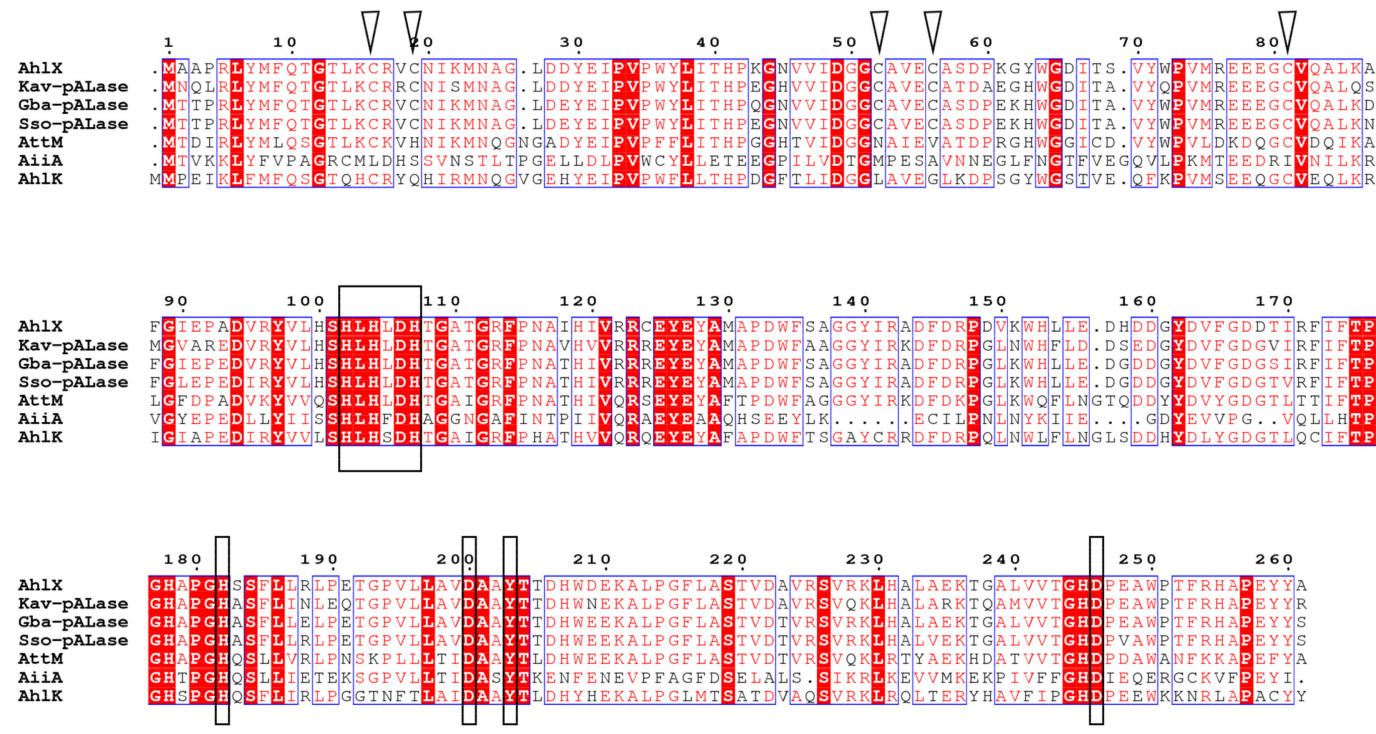

Figure 2. Multiple sequence alignment between AhlX and other AHL lactonases. AhlX, AHL lactonase from S. salaries A10339; Kav-pALase (SFC91482.1), predicted AHL lactonase from the halophilic bacterium K. avicenniae; Gba-pALase (WP_035471581.1), predicted AHL lactonase from the halophilic bacterium Gammaproteobacteria bacterium MFB021; Sso-pALase (WP_075571152.1), predicted AHL lactonase from the halophilic bacterium S. socius; AttM (AAL13075.1), AHL lactonase from A. tumefaciens; AiiA (AAF62398.1), AHL lactonase from Bacillus sp. 240 B1; and AhIK (AAO47340.1), AHL lactonase from K. pneumoniae. The conserved residues that are crucial for the activity of AHL lactonases are indicated with black rectangles. The conserved cystines present in Kav-pALase, AhlX, Gba-pALase and Sso-pALase are indicated by black triangles.
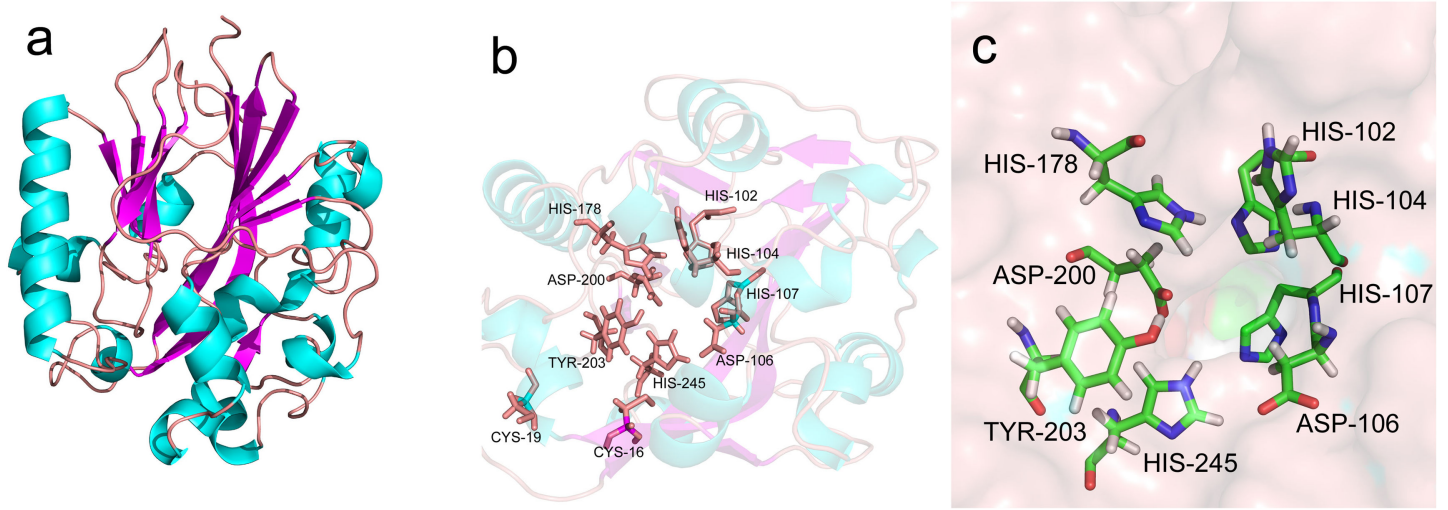

Figure 3. In silico modeling of the structure of AhlX. The 3D-structure modeling of AhlX was performed using the Protein Function and Structure Prediction server, I-TASSER (http://zhanglab.ccmb.med.umich. edu/I-TASSER) [24] and illustrated using Swiss-PdbViewer. (a) The modeled 3D structure of AhlX; and (b) predicted functional sites in AhlX. Previously, identified important active site residues of AHL lactonase $\left(\mathrm{H}^{102}, \mathrm{H}^{104}, \mathrm{D}^{106}, \mathrm{H}^{107}, \mathrm{H}^{178}, \mathrm{D}^{200}, \mathrm{Y}^{203}\right.$ and $\left.\mathrm{H}^{245}\right)$ and the distinct $\mathrm{C}^{16} X X \mathrm{C}^{19}$ motif identified in this study are illustrated. (c) A partial enlarged view of predicted functional sites in AhlX. The protein was shown as surface model, and catalytic residues were shown as stick model, respectively. 


\subsection{Characterization of 3OC8-HSL Degradation by AhlX}

To validate the activity of AhlX, ahlX was cloned into the vector pET28a and expressed in the host strain E. coli BL21 (DE3). The recombinant E. coli strain showed clear AHL-degrading activity as detected through the A. tumefaciens NT1 bioassay method (Figure 4a). Subsequently, AhlX was purified from the recombinant $E$. coli strain, in which a considerable amount of soluble protein was obtained after induction at $30^{\circ} \mathrm{C}$ for $16 \mathrm{~h}$ with $0.2 \mathrm{mmol} / \mathrm{L}$ IPTG (Figure $4 \mathrm{~b}$ ). AhlX was further purified by one-step $\mathrm{Ni}^{2+}$-NTA affinity purification. SDS-PAGE analysis showed that AhlX exhibited a clear band at a molecular mass of approximately $30 \mathrm{kDa}$, corresponding to its predicted molecular mass (Figure 4b).

a

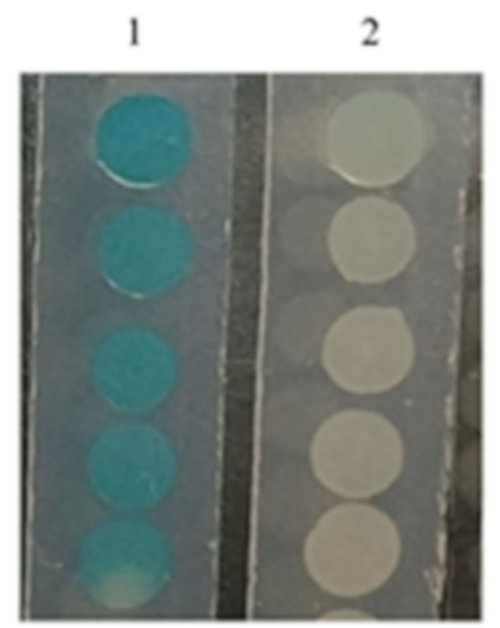

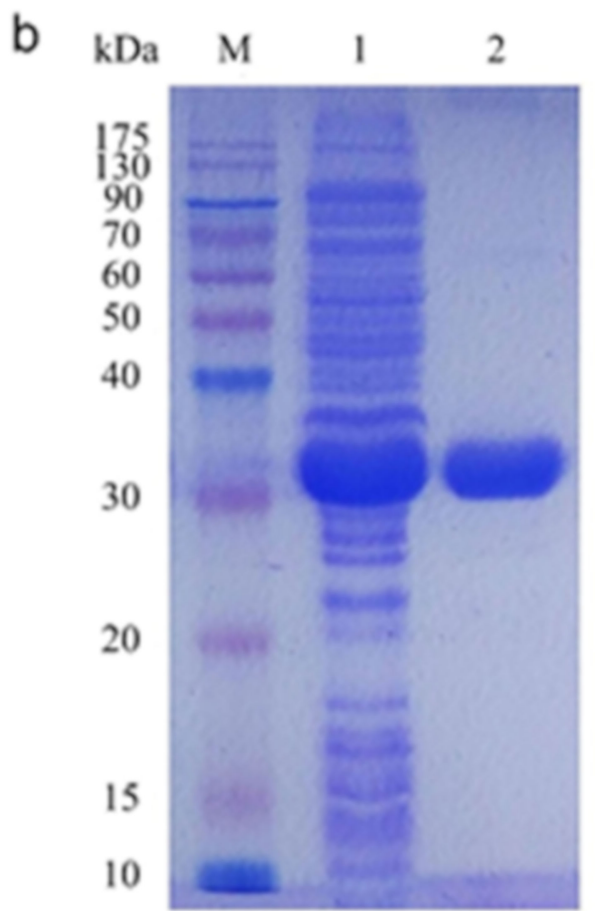

Figure 4. Degradation of 3OC8-HSL by AhlX and purification of recombinant AhlX. (a) Degradation of 3OC8-HSL by E. coli BL21-pET28a-ahlX. Lane 1, $0.5 \mu \mathrm{mol} / \mathrm{L}$ of the 3OC8-HSL sample; Lane 2, 3OC8-HSL degradation product generated by AhlX; (b) SDS-PAGE analysis of AhlX expressed in E. coli BL21 (DE3) and purified recombinant AhlX. Lane M, protein marker; Lane 1, supernatant of induced E. coli cells harboring pET28a-ahlX; Lane 2, purified recombinant AhlX.

To determine whether AhlX works as an AHL lactonase, the substrate 3OC8-HSL was used to test the activity of AhlX, and the resulting degradation product was further analyzed. As shown in Figure 5a, the partial degradation of 3OC8-HSL by AhlX yielded two peaks with the retention times of 4.64 and $3.2 \mathrm{~min}$ by HPLC. Since the undegraded 3-OC8-HSL gave a retention time of $4.64 \mathrm{~min}$, the $3.2 \mathrm{~min}$ peak is likely a 3OC8-HSL degradation product generated by AhIX. This peak was collected and sent for mass spectrometry analysis. As shown in Figure 5c, the 3.2 min peak collected from HPLC had an $\mathrm{m} / \mathrm{z}$ (mass-to-charge ratio) of 258.12, whereas the 3OC8-HSL had an $\mathrm{m} / \mathrm{z}$ of 240.12 (Figure $5 \mathrm{~b}$ ). These results indicated that the AhlX-mediated degradation of 3OC8-HSL generated a product with an increased $\mathrm{m} / \mathrm{z}$ of 18 (Figure $5 \mathrm{~d}$ ), in line with the property of 3OC8-HS, which is generally produced by AHL lactonase from 3OC8-HSL. These results, in combination with aforementioned bioinformatics analysis, suggest that AhlX is a typical AHL lactonase. 


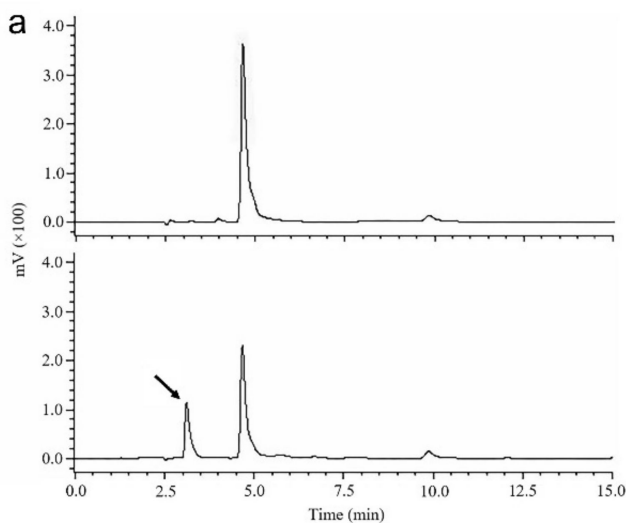

d

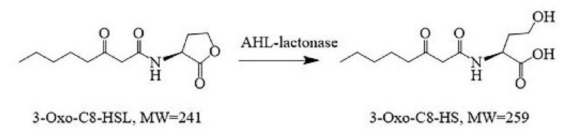

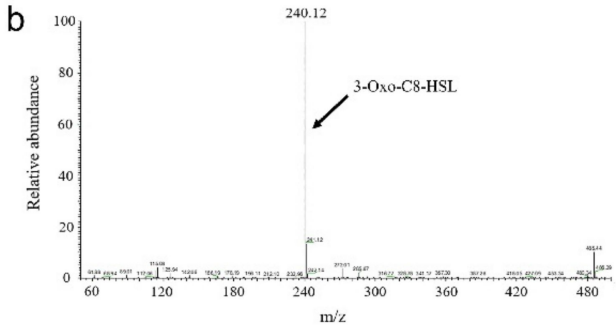

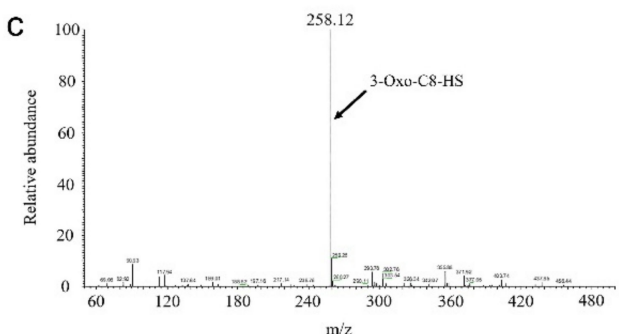

Figure 5. HPLC and LC-MS analysis of 3OC8-HSL the degradation product generated by AhlX. (a) HPLC analysis of 3OC8-HSL and its degradation product generated by AhlX. The upper panel shows the HPLC profile of 3OC8-HSL, while the lower panel shows the HPLC profile with the 3OC8-HSL degradation product generated by AhlX. The arrow indicates the expected degradation product at a retention time of $3.2 \mathrm{~min}$. (b) LC-MS profile of 3OC8-HSL. The arrow indicates 3OC8-HSL with an $m / z$ of 240.12. (c) LC-MS analysis of the 3OC8-HSL degradation product generated by AhlX. The arrow indicates the expected degradation product with an $\mathrm{m} / \mathrm{z}$ of 258.12. (d) Schematic illustration of the mechanism of 3OC8-HSL degradation by AHL lactonase.

\subsection{Biochemical Characterization of AhlX}

AhlX showed considerable activity at temperatures from 10 to $60{ }^{\circ} \mathrm{C}$, with the optimum activity observed at $40{ }^{\circ} \mathrm{C}$. The enzyme exhibited approximately $50 \%$ of its maximal activity at $10{ }^{\circ} \mathrm{C}$ and approximately $40 \%$ at $60^{\circ} \mathrm{C}$ (Figure 6a). AhlX displayed very good activity at pH values of 7.0 to 9.0, but its activity decreased sharply when the $\mathrm{pH}$ decreased below 6.0 (Figure $6 \mathrm{~b}$ ). These results suggest that AhlX is sensitive to an acidic catalysis environment.

As shown in Figure 6c, AhlX was activated by several divalent metal ions, including $\mathrm{Mg}^{2+}, \mathrm{Mn}^{2+}$, $\mathrm{Co}^{2+}, \mathrm{Ni}^{2+}$ and $\mathrm{Zn}^{2+}$. In the presence of $1 \mathrm{mmol} / \mathrm{L} \mathrm{Mn}^{2+}, \mathrm{Co}^{2+}$ and $\mathrm{Zn}^{2+}$, the activity of AhlX was increased by over 1 -fold, whereas the addition of $1 \mathrm{mmol} / \mathrm{L} \mathrm{Ni}^{2+}$ increased the activity by over 2 -fold. However, $\mathrm{Ca}^{2+}$ did not affect the activity of $\mathrm{AhlX}$, and $\mathrm{Fe}^{2+}$ and $\mathrm{Cu}^{2+}$ significantly inhibited the activity of AhlX. In addition, $1 \mathrm{mmol} / \mathrm{L}$ EDTA also decreased the activity of AhlX by approximately $50 \%$.

AhlX showed activity towards a wide spectrum of substrates. As shown in Figure 6d, AhlX exhibited over $60 \%$ activity towards all of the tested AHL-typed substrates, including C4-HSL, C6-HSL, C8-HSL, C10-HSL, C12-HSL, C14-HSL, 3OC6-HSL, 3OC8-HSL, 3OC12-HSL and 3OC14-HSL. Nevertheless, AhlX appeared to perform better against 3-oxo-AHLs and displayed the best activity towards the substrates C6-HSL, 3OC6-HSL and 3OC8-HSL.

Notably, AhlX displayed extraordinary temperature stability. As shown in Figure 7a, after incubation at 0 to $60{ }^{\circ} \mathrm{C}$ for $30 \mathrm{~min}$, the activity of AhlX showed nearly no change. In a thermal stability test for an extended period of time, AhlX maintained approximately $97 \%$ activity after incubating at $25{ }^{\circ} \mathrm{C}$ for 12 days (Figure $7 \mathrm{~b}$ ) and over $40 \%$ activity after incubating at $60{ }^{\circ} \mathrm{C}$ for $6 \mathrm{~h}$ (Figure $7 \mathrm{c}$ ). Moreover, as shown in Figure 7d, AhlX exhibited strong salt tolerance, with over $80 \%$ of the activity of AhlX remaining when $15 \% \mathrm{NaCl}$ was added into the reaction mixture. Specifically, at the high $\mathrm{NaCl}$ concentration of $25 \%, 60 \%$ of AhlX activity was retained. 
a

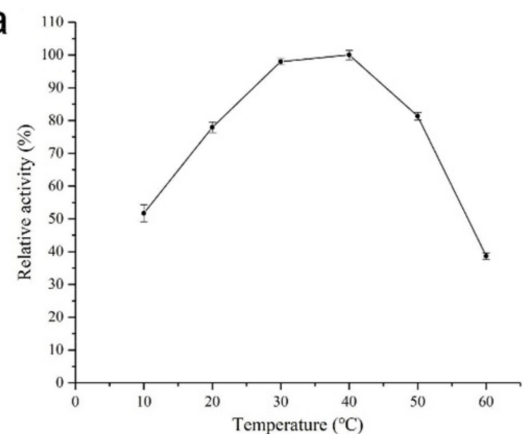

C

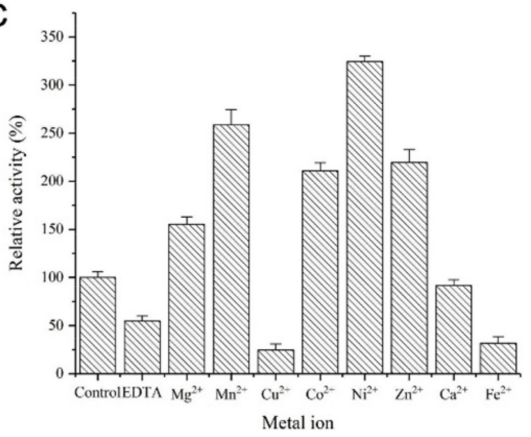

b

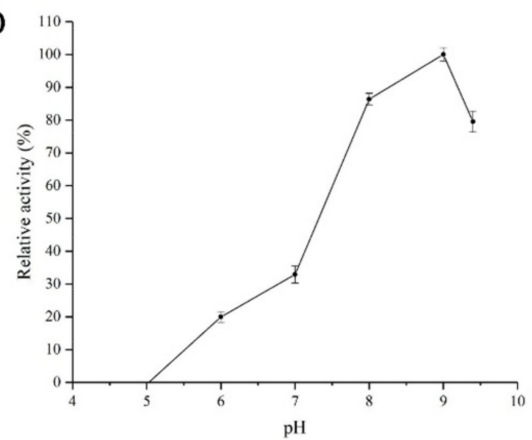

d

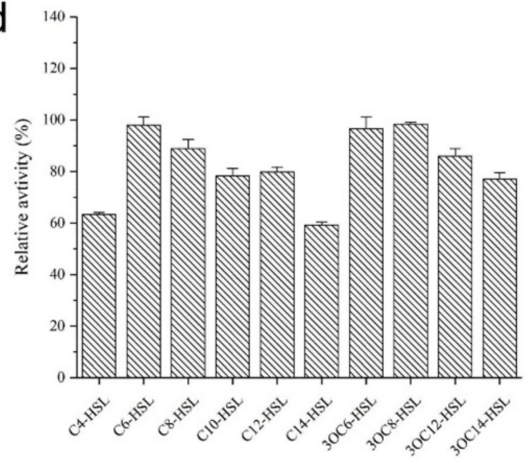

Figure 6. Biochemical characterization of AhlX. (a) Effects of temperature on the activity of AhlX. (b) Effects of $\mathrm{pH}$ on the activity of AhlX. (c) Effects of different divalent cations on the activity of AhlX. (d) Substrate specificity of AhlX.
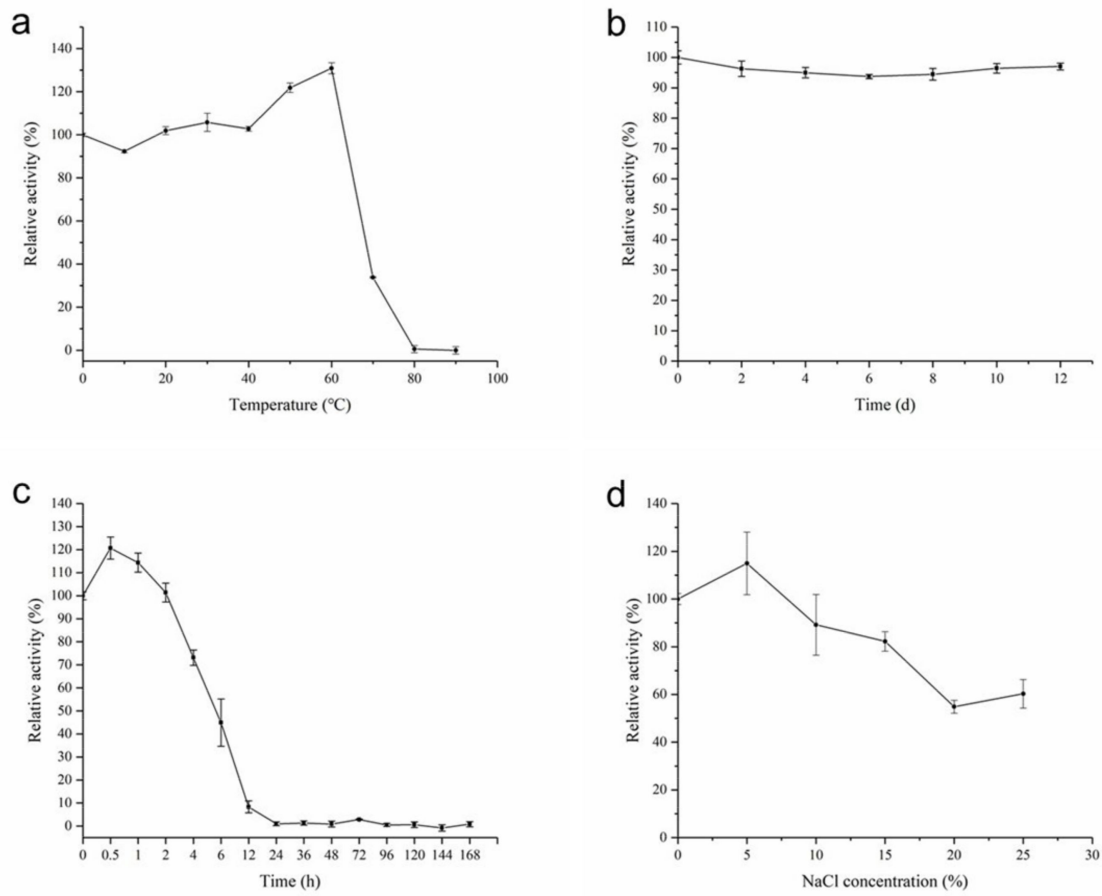

Figure 7. The temperature stability and salt tolerance of AhlX. (a) The thermal stability of AhlX at different temperatures. (b) The time-dependent thermal stability of AhlX at $25^{\circ} \mathrm{C}$. (c) The time-dependent thermal stability of AhlX at $60^{\circ} \mathrm{C}$. (d) Effect of different $\mathrm{NaCl}$ concentrations on the activity of AhlX. 


\subsection{Quenching the E. carotovora Infection by AhlX}

AHL lactonases have been suggested to play important roles in quenching bacterial diseases $[9,10,26]$. E. carotovora SCG1 is a bacterial pathogen that generally elicits the so-called bacterial soft disease in several important vegetables, including potato and cabbage. It has been reported that the infection process of $E$. carotovora SCG1 strongly relies on the expression of pathogenic genes controlled by AHL signaling [27]. To assess the activity of AhlX in the practical control of bacterial diseases, we tested the ability of AhlX to quench E. carotovora infection in potato.

E. coli is generally used as model organism to test the potential of in vivo function of gene. Therefore, we tested the activity of a recombinant E. coli strain in combating E. carotovora infection. The E. coli strain BL21(DE3) expressing AhlX was co-cultured with E. carotovora SCG1 on potato slices. As shown in Figure $8 \mathrm{a}$, after incubating at $30^{\circ} \mathrm{C}$ for $40 \mathrm{~h}$, no obvious soft rot symptom was observed on the potato slices inoculated with both strains, whereas clear symptoms were observed on the potato slices inoculated with only E. carotovora SCG1 or E. carotovora SCG1-E. coli BL21 (no AhlX expression). These results suggest that the recombinant E. coli strain expressing AhIX inhibited E. carotovora infection.
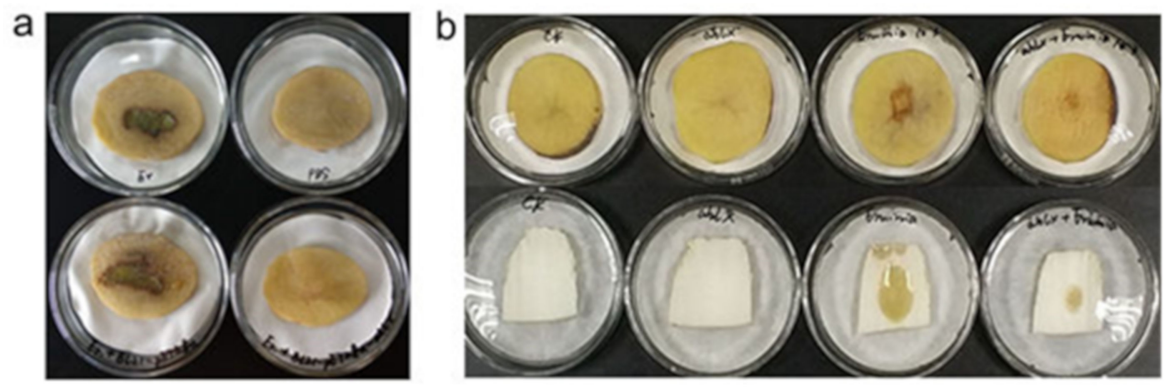

Figure 8. The inhibitory effect of AhlX toward the soft rot infectious disease caused by E. carotovora SCG1. (a) The inhibitory effect of E. coli BL21-pET28a-ahlX towards the soft rot infectious disease caused by $E$. carotovora SCG1. The surface-sterilized potato slices were inoculated separately with $E$. carotovora SCG1 (upper left), Phosphate Buffered Saline (PBS) $(\mathrm{pH}=7.4)$ (upper right), the E. carotovora SCG1 and E. coli BL21-pET28a mixture (bottom left), or the E. carotovora SCG1 and E. coli BL21-pET28a-ahlX mixture (bottom right). (b) The inhibitory effect of spray-dried AhIX on soft rot caused by E. carotovora SCG1. The surface-sterilized potato slices and Chinese cabbage stalks were treated separately with PBS $(\mathrm{pH}=7.4)$, spray-dried AhlX, E. carotovora SCG1, E. carotovora SCG1 and spray-dried AhlX (from left to right).

The high temperature stability of AhlX suggested that it should have a potential to be used as an in vitro agent for AHL quenching and bacterial disease control. We further tested the activity of AhlX in vitro against E. carotovora infection. To mimic the commercial enzyme production process, we generated AhlX powder directly from the crude lysate of E. coli cells expressing AhlX through an industrial spray-drying process. The cell lysate of the E. coli culture expressing AhlX was first heated at $60{ }^{\circ} \mathrm{C}$ for $30 \mathrm{~min}$ to remove the heat-sensitive proteins. Subsequently, a spray-drying process at $80-120^{\circ} \mathrm{C}$ was used to generate the dried AhlX powder. The spray-dry process did not significantly alter the activity of AhlX, and the total recovery rate of the AhlX activity from the original lysate supernatant reached $84.06 \%$, suggesting that the AhlX production process was successful (Table 1). As shown in Figure 8 b, the spray-dried AhlX powder clearly reduced the E. carotovora-induced infectious symptoms of soft rot on potato slices and Chinese cabbage stalks after incubation at $30^{\circ} \mathrm{C}$ for $20 \mathrm{~h}$. 
Table 1. The activity of AhlX prepared by different methods.

\begin{tabular}{ccccc}
\hline Purification Step & Total Protein (mg) & $\begin{array}{c}\text { Specific Activity } \\
\text { (Units/mg) }\end{array}$ & $\begin{array}{c}\text { Total Activity } \\
\text { (Units) }\end{array}$ & $\begin{array}{c}\text { Enzyme Activity } \\
\text { Recovery (\%) }\end{array}$ \\
\hline Lysate supernatant & 507.06 & 108.89 & $5.52 \times 10^{4}$ & 100 \\
Heat treatment & 247.35 & 223.76 & $5.53 \times 10^{4}$ & 100.24 \\
Heat treatment + Spray drying & 203.36 & 228.25 & $4.64 \times 10^{4}$ & 84.06 \\
\hline
\end{tabular}

\section{Discussion}

In this study, we characterized the lactonase AhlX from the marine bacterium S. salaries MCCC1A01339. Members of the Salinicola genus are halophilic bacteria. Halophiles grow in the hyper-saline conditions (up to $30 \% \mathrm{NaCl}$ ) and have attracted particular research interest for their ability to resist osmotic stress and salt-induced denaturation. Therefore, these bacteria and their enzymes have great biotechnological potential with respect to environmental remediation, enzyme development and ectoine production $[28,29]$. To date, QS signaling molecules AHLs have been detected in several halophilic bacteria, including Chromohalobacter sp., Cobetia sp., Halomonas sp., Halotalea sp., Kushneria sp., Modicisalibacter sp. and Salinicola sp. [30,31]. However, lactonases, AHL-quenching enzymes, have never been characterized within this group of bacteria. Therefore, the characterization of AhlX should provide a good reference for further physiological and biotechnological studies of AHL quenching by halophilic species.

AhlX shares distinct amino acid sequence with other well-characterized AHL lactonase. AhlX and AHL lactonases to be phylogenetically close, including Gba-pALase, Sso-pAlase and Kav-pALase, all contain five cysteines, residues that are not conserved in other well-characterized AHL lactonases such as AiiA from Bacillus sp. 240 B1 and AiiM from Microbacterium testaceum. The CXXC motif is suggested to be important in redox sensing-dependent protein function [32]. Despite the detailed roles of these residues being unknown, the conservation of these residues associated with redox-sensing in AhlX, Gba-pALase, Sso-pAlase and Kav-pALase enabled us to envision their different catalytic mechanisms. While it is unclear whether these cysteines are the hallmark of AhlX phylogenetically close group, future studies can help to elucidate their detailed functions.

AhlX retained over $97 \%$ activity after incubating at $25^{\circ} \mathrm{C}$ for 12 days $(288 \mathrm{~h})$ and at $60^{\circ} \mathrm{C}$ for $2 \mathrm{~h}$, with even approximately $44 \%$ of its activity retained at $60^{\circ} \mathrm{C}$ for $6 \mathrm{~h}$. Therefore, AhlX is one of the most stable AHL lactonases identified to date. It has been previously reported that AiiA could maintain $>99 \%$ activity at $21^{\circ} \mathrm{C}$ for $240 \mathrm{~h}$, yet its activity went down sharply after incubating at $45^{\circ} \mathrm{C}$ for $2 \mathrm{~h}$ [33]. the $N$-acylhomoserine lactonase from the thermophilic bacterium Geobacillus caldoxylosilyticus YS- 8 was previously shown to retain full activity after pre-incubating at $40{ }^{\circ} \mathrm{C}$ for $3 \mathrm{~h}$. However, the activity of this enzyme decreased to approximately $50 \%$ of its maximum when incubated at $60{ }^{\circ} \mathrm{C}$ for $2 \mathrm{~h}$ [34]. Although the mechanism associated with the high stability of AhlX is currently unknown, this property makes it an excellent candidate for use as an in vitro preventive and curative agent for bacterial disease. As shown in Figure 8b, the AhlX powder made through a spray-drying process at $80-120^{\circ} \mathrm{C}$ maintained high activity and performed well at preventing E. carotovora infection. Further work should focus on testing the activity of AhlX activity against other pathogenic bacteria to boost its commercial use. AhlX showed strong salt tolerance. To the best of our knowledge, AhIX is the first AHL lactonase characterized that can tolerate $25 \% \mathrm{NaCl}$. In past decades, pathogenic halophilic bacteria have been identified as the culprits of numerous diseases. For example, Halomonas sp. was recently recognized as a human pathogen implicated in infections and contamination in a dialysis center [35]. Since AHL-dependent QS has been implicated in regulating virulence gene expression in many bacterial pathogens, it is easy to envision a connection between AHLs and the pathogenicity of halophiles. Therefore, it is our expectation that future applications of AhlX will involve the quenching of AHLs produced by halophilic bacterial pathogens to control diseases.

AhlX also shares several similar properties with other well-characterized AHL lactonases. AhlX showed optimal activity at $\mathrm{pH} 7.0-8.0$ and $40^{\circ} \mathrm{C}$ (Figure 2). These properties resemble those of other 
reported AHL lactonases, such as AiiA from Bacillus sp. 240 B1 [33] and AidC from Chryseobacterium sp. strain StRB126 [20]. Notably, AhlX showed broad substrate specificity, with over $60 \%$ relative activity observed toward all tested AHLs, with or without 3-oxo substitution (Figure 6d). The previous reports also showed that AiiA from Bacillus sp. 240B1 [33] and AiiM from Microbacterium testaceum [36] could degrade a wide variety of AHLs. While different bacteria growing in communities tend to use different types of AHLs to control their QS-dependent behavior or survival, the broad substrate specificity of AhlX should make $S$. salaries highly competitive in the niches it occupies.

In summary, in this study, we identified and characterized a highly stable AHL lactonase, AhlX, with a unique amino acid composition. AhlX maintained high activity during its preparation at high temperature using an industrial used spray-drying process, indicating the possibility for its low-cost production at a large scale. The spray-dried AhlX powder demonstrated control of E. carotovora infection in vitro, suggesting its potential for the further application in bacterial disease control.

\section{Materials and Methods}

\subsection{Bacterial Strains and Chemicals}

E. coli BL21(DE3) was used to express the AhlX protein driven by the pET28a vector. The strain A. tumefaciens NT1 expressing an AHL-regulated LacZ was used to evaluate the AHL activity with the bioassay method described by Dong et al. [9]. E. carotovora SCG1 was provided by Prof. Ziduo Liu (HuaZhong Agricultural University, Wuhan, China) and used to test the quenching activity of AhlX for bacterial disease.

The AHLs used in this study included N-butyryl-L-Homoserine lactone (C4-HSL), $\mathrm{N}$-hexanoyl-L-homoserine lactone (C6-HSL), N-octanoyl-L-homoserine lactone (C8-HSL), $\mathrm{N}$-decanoyl-L-homoserine lactone (C10-HSL), N-dodecanoyl-L-homoserine lactone (C12-HSL), $\mathrm{N}$-tetradecanoyl-L-homoserine lactone (C14-HSL), N-(3-oxohexanoyl)-L-homoserine lactone (3OC6-HSL), $\mathrm{N}$-(3-oxooctanoyl)-L-homoserine lactone (3OC8-HSL), N-(3-oxododecanoyl)-L-homoserine lactone (3OC12-HSL), and N-(3-oxo-tetradecanoyl)-L-homoserine lactone (3OC14-HSL) and were purchased from Cayman Chemical Company (Ann Arbor, MI, USA). Other chemicals, if not specified, were purchased from Sinopharm Group Co. Ltd. (Shanghai, China).

\subsection{Genome Sequencing and Bioinformatic Analysis}

The draft genome sequence of $S$. salaries MCCC1A01339 was obtained using an Illumina MiSeq by Majorbio (Shanghai, China). Gene prediction was performed using Glimmer 3.02 (http://www. cbcb.umd.edu/software/glimmer, the Center for Computational Biology at Johns Hopkins University, Baltimore, MD, USA), and gene annotation was performed by aligning the predicted coding sequences of each gene to those sequences in the non-redundant gene databases (https://www.ncbi.nlm.nih.gov/) and string (http://string-db.org/) and the GO database (http://www.geneontology.org/page/go-database) by Blast analysis. Multiple sequence alignment analysis of AHL lactonases was performed using ClustalX implemented in BioEdit Version 7.0 .5 (http://www.mbio.ncsu.edu/bioedit/bioedit.html, Tom Hall; Ibis Therapeutics, Carlsbad, CA, USA) and was illustrated using ESPript Version 3.0 (http: //espript.ibcp.fr/ESPript/cgi-bin/ESPript.cgi, "Retroviruses and Structural Biochemistry" research team of the "Molecular Microbiology and Structural Biochemistry" laboratory (UMR5086 CNRS/Lyon University). Lyon Cedex 07, France) [37]. The phylogenetic tree of AHL lactonases was generated using the neighbor-joining method and was illustrated using Tree-view X Version 0.5.0 (http:// taxonomy.zoology.gla.ac.uk/rod/treeview.html, Roderic D. M. Page, UK). The 3D-structure modeling of AhlX was performed using the Protein Function and Structure Prediction server, I-TASSER (http: //zhanglab.ccmb.med.umich.edu/I-TASSER, Yang Zhang's Research Group, University of Michigan, Ann Arbor, MI, USA) and was illustrated using Swiss-Pdb Viewer Version 4.1 (Swiss Institute of Bioinformatics, Lausanne, Switzerland). 


\subsection{Expression and Purification of AhlX}

To obtain the recombinant AhlX, the ahlX gene was PCR amplified using the forward primer GACGTGCATATGGCCGCTCCACGTCTCTATATG and the reverse primer GCTGAATTCTCAAGC GTAGTATTCCGGGGC using standard procedures. The amplified DNA fragment was subsequently ligated into the expression vector pET-28a to generate the plasmid pET28a-ahlX, which was subsequently transformed into the E. coli DH5 $\alpha$ plasmid extraction and sequence analysis. Subsequently, a pET28a-ahlX clone with the correct sequence was transformed into the protein expression host E. coli BL21(DE3). To express AhlX, $0.2 \mathrm{mmol} / \mathrm{L}$ IPTG was added to the culture at an $\mathrm{OD}_{600}$ of $\sim 0.6$, and the culture was further grown at $30{ }^{\circ} \mathrm{C}$ for $16 \mathrm{~h}$. AhlX was purified using $\mathrm{Ni}^{2+}-\mathrm{NTA}$ affinity chromatography following the manufacturer's protocol (GE Healthcare, Uppsala, Sweden). Gel filtration chromatography was subsequently performed to remove salt ions from the protein sample using Sephadex ${ }^{\mathrm{TM}}$ G-25M (GE Healthcare, Uppsala, Sweden). For the detection and analysis of the expressed and purified AhlX, 15\% $(v / v)$ sodium dodecyl sulfate-denatured poly-acrylamide gel electrophoresis (SDS-PAGE) analysis was performed. The purified AhlX protein was stored in $-80{ }^{\circ} \mathrm{C}$ for later use. Spray-drying of AhlX was performed using a JT-6000Y spray drier (Hangzhou Jtone Electronic Co.Ltd., Hangzhou, China) with an air speed of $100 \mathrm{~L} / \mathrm{min}$, an air inlet temperature of $120^{\circ} \mathrm{C}$ and an air outlet temperature of $80^{\circ} \mathrm{C}$.

\subsection{Standard Activity Assay of AhlX}

The standard AhlX activity assay was essentially performed as described by Huang et al. [14]. Briefly, $5 \mu \mathrm{L}$ of purified AhlX was added to $400 \mu \mathrm{L}$ of $10 \mathrm{mmol} / \mathrm{L}$ phosphate buffer (PB, $\mathrm{pH} 8.0$ ) containing $0.83 \mathrm{mmol} / \mathrm{L} 3 \mathrm{OC} 8$-HSL substrate and incubated at $30{ }^{\circ} \mathrm{C}$ for $30 \mathrm{~min}$. The reaction was terminated with SDS solution at a final concentration of $2 \%$ and was detected by high-performance liquid chromatography (HPLC) on a Diamonsil ${ }^{\circledR} \mathrm{C} 18$ column $(4.6 \times 250 \mathrm{~mm}, 5 \mu \mathrm{m}$, Dikma, Beijing, China) with a mobile phase of $\mathrm{C}_{2} \mathrm{H}_{3} \mathrm{~N}: \mathrm{HCOOH}: \mathrm{H}_{2} \mathrm{O}(50:$ 0.2: 49.8$)$ at $1 \mathrm{~mL} / \mathrm{min}$. The detection wavelength was set to $201 \mathrm{~nm}$.

\subsection{Determination of the Mechanism of AHL Degradation by AhlX}

To degrade 3OC8-HSL, purified AhlX (final concentration of $18.32 \mu \mathrm{g} / \mathrm{mL}$ ) was mixed with 3OC8-HSL (final concentration of $2.0 \mathrm{mmol} / \mathrm{L}$ ) in $2.0 \mathrm{~mL}$ of $10 \mathrm{mmol} / \mathrm{L}$ potassium phosphate buffer ( $\mathrm{pH}$ 8.0) and incubated at $30^{\circ} \mathrm{C}$ for $7 \mathrm{~h}$. The reaction mixture was then extracted with an equal volume of ethyl acetate twice, and the product in the organic layer was subsequently collected and concentrated with a vacuum centrifugal concentrator RVC 2-25 CD plus (Christ, Germany). A similar procedure was also used for the reaction mixture without AhlX.

For subsequent chromatographic analysis, each extract was then dissolved in methanol $(200 \mu \mathrm{L})$, with $8 \mu \mathrm{L}$ subsequently analyzed by HPLC-MS (Thermo Scientific ${ }^{\mathrm{TM}}$ LCQ Fleet ${ }^{\mathrm{TM}}$, Thermo Fisher Scientific, Waltham, MA, USA) on a Diamonsil ${ }^{\circledR} \mathrm{C} 18(250 \times 4.6 \mathrm{~mm}, 5 \mu \mathrm{m})$ column with a mobile phase of $\mathrm{C}_{2} \mathrm{H}_{3} \mathrm{~N}: \mathrm{HCOOH}: \mathrm{H}_{2} \mathrm{O}(50: 0.2: 49.8)$ at $1 \mathrm{~mL} / \mathrm{min}$, a detection wavelength of $201 \mathrm{~nm}$ and the column temperature set at $30^{\circ} \mathrm{C}$. Samples ionized by negative electrospray were used for MS analysis with a mass range of $m / z 50$ to 500 scanned under the following conditions: sheath gas flow rate of $75 \mathrm{arb}$, Aux gas flow rate of $20 \mathrm{arb}$, sweep gas flow rate of $0 \mathrm{arb}$, I spray voltage of $5 \mathrm{kV}$, capillary temperature of $300^{\circ} \mathrm{C}$, capillary voltage of $-10 \mathrm{~V}$ and a tube lens compensation voltage of $-125 \mathrm{~V}$.

\subsection{Biochemical Characterization of AhlX}

The optimum temperature and $\mathrm{pH}$ for AhlX activity toward 3-OC8-HSL, was assessed at different temperature $\left(10\right.$ to $\left.60{ }^{\circ} \mathrm{C}\right)$ and different $\mathrm{pH}$ values $\left(\mathrm{KH}_{2} \mathrm{PO} 4-\mathrm{K}_{2} \mathrm{HPO} 4\right.$ buffer for $\mathrm{pH}$ 5.0-9.4) under the standard conditions. The effect of metal ions on AhlX activity was evaluated by determining the relative enzyme activity using 3OC8-HSL as substrate in the presence of $1 \mathrm{mmol} / \mathrm{L}$ concentrations of different metal ions. To characterize the substrate specificity of AhlX, homoserine lactones with variable chain length and modifications, including C4-HSL, C6-HSL, C8-HSL, C10-HSL, C12-HSL, 
C14-HSL, 3OC6-HSL, 3OC8-HSL, 3OC12-HSL, and 3OC14-HSL were used as substrates to test its activity. To measure the stability of AhlX at different temperatures, the enzyme was incubated at 0 to $90{ }^{\circ} \mathrm{C}$ for $30 \mathrm{~min}$, after which the residual activity was measured under the standard conditions. Furthermore, to measure the time-dependent thermal stability of AhlX, its residual activity was tested after incubation at $60^{\circ} \mathrm{C}$ for $0.5,1,2,4,6,12$ and $24 \mathrm{~h}$, and at $25^{\circ} \mathrm{C}$ at 48 -h intervals from 0 to $288 \mathrm{~h}$. To determine the salt tolerance of $\mathrm{AhlX}, 0-25 \% \mathrm{NaCl}$ was added into the reaction mixture and its residual activity was measured.

\subsection{Quenching the E. carotovora Infection by AhlX}

To test the inhibitory effect of the recombinant strain E. coli BL21-pET28a-ahlX towards the soft rot infectious disease caused by E. carotovora, $7.5 \mu \mathrm{L}$ of an overnight culture of E. carotovora $\left(7.2 \times 10^{5} \mathrm{CFU} / \mathrm{mL}\right)$ and $7.5 \mu \mathrm{L}$ of an overnight culture of E. coli BL21-pET28a-ahlX $\left(1.8 \times 10^{6} \mathrm{CFU} / \mathrm{mL}\right)$ was mixed evenly and inoculated in the center of the potato slices. As a control, $7.5 \mu \mathrm{L}$ of E. carotovora and E. coli BL21-pET28a-ahlX and $7.5 \mu \mathrm{L} 10 \mathrm{mmol} / \mathrm{L}$ PBS (pH 7.4) were mixed with $7.5 \mu \mathrm{L}$ of PBS and inoculated in the center of the potato slices. After incubating at $30^{\circ} \mathrm{C}$ for $40 \mathrm{~h}$, the presence of soft rot symptom was assessed.

To determine the ability of spray-dried AhlX powder to quench the E. carotovora infection, $1.5 \mathrm{mg}$ of spray-dried AhlX powder was dissolved into $150 \mu \mathrm{L}$ PBS buffer and immediately spread onto potato slices and Chinese cabbage stems, after which $1 \mu \mathrm{L}$ of an overnight culture of E. carotovora $\left(3.6 \times 10^{5} \mathrm{CFU} / \mathrm{mL}\right)$ was inoculated. The potato slices and Chinese cabbage stems treated with only $10 \mathrm{mmol} / \mathrm{L} \mathrm{PBS} \mathrm{(pH} \mathrm{7.4),}$ spray-dried AhlX $(1 \mathrm{~g} / \mathrm{L})$ or E. carotovora $\left(3.6 \times 10^{5} \mathrm{CFU} / \mathrm{mL}\right)$ were used as controls. After incubating at $30^{\circ} \mathrm{C}$ for $20 \mathrm{~h}$, the presence of soft rot symptom was assessed.

\subsection{Nucleotide Sequence Accession Number}

The nucleotide sequences of ahlX gene, 16S rDNA of S. salaries MCCC1A01339, and 23S rDNA of S. salaries MCCC1A01339 have been deposited in the GenBank database under the accession numbers KY783591, KY783592 and KY783593, respectively. The draft genome of S. salaries MCCC1A01339 was deposited in the BioProject database under the ID PRJNA379806.

Author Contributions: X.C. and H.W. supervised the research and revised the manuscript; P.L. designed the experiments and wrote the manuscript; Y.C. performed the experiments and analyzed the data; Z.S. provided the strain S. salaries MCCC1A01339; J.C. and J.P. revised the manuscript; and J.W and Q.G. contributed to part of the characterization and assay of AhlX.

Funding: This study was supported by National Natural Science Foundation of China (Grant No. 41306142).

Acknowledgments: We are very grateful to Ziduo Liu (HuaZhong Agricultural University), who kindly provided us A. tumefaciens strain NT1 and E. carotovora strain SCG1.

Conflicts of Interest: The authors declare no conflicts of interest.

\section{References}

1. Bassler, B.L.; Greenberg, E.P.; Stevens, A.M. Cross-species induction of luminescence in the quorum-sensing bacterium Vibrio harveyi. J. Bacteriol. 1997, 179, 4043-4045. [CrossRef]

2. Lang, J.; Faure, D. Functions and regulation of quorum-sensing in Agrobacterium tumefaciens. Front. Plant Sci. 2014, 5, 14. [CrossRef] [PubMed]

3. Duerkop, B.A.; Varga, J.; Chandler, J.R.; Peterson, S.B.; Herman, J.P.; Churchill, M.E.; Parsek, M.R.; Nierman, W.C.; Greenberg, E.P. Quorum-sensing control of antibiotic synthesis in Burkholderia thailandensis. J. Bacteriol. 2009, 191, 3909-3918. [CrossRef]

4. Allison, D.G.; Ruiz, B.; SanJose, C.; Jaspe, A.; Gilbert, P. Extracellular products as mediators of the formation and detachment of Pseudomonas fluorescens biofilms. FEMS Microbiol. Lett. 1998, 167, 179-184. [CrossRef] [PubMed]

5. De Kievit, T.R.; Iglewski, B.H. Bacterial quorum sensing in pathogenic relationships. Infect. Immun. 2000, 68, 4839-4849. [CrossRef] [PubMed] 
6. Waters, C.M.; Bassler, B.L. Quorum sensing: cell-to-cell communication in bacteria. Annu. Rev. Cell Dev. Biol. 2005, 21, 319-346. [CrossRef] [PubMed]

7. Parsek, M.R.; Greenberg, E.P. Acyl-homoserine lactone quorum sensing in gram-negative bacteria: a signaling mechanism involved in associations with higher organisms. Proc. Natl. Acad. Sci. USA 2000, 97, 8789-8793. [CrossRef] [PubMed]

8. Andersson, R.A.; Eriksson, A.R.; Heikinheimo, R.; Mae, A.; Pirhonen, M.; Koiv, V.; Hyytiainen, H.; Tuikkala, A.; Palva, E.T. Quorum sensing in the plant pathogen Erwinia carotovora subspcarotovora: the role of expR(Ecc). Mol. Plant-Microbe Interact. 2000, 13, 384-393.

9. Dong, Y.H.; Xu, J.L.; Li, X.Z.; Zhang, L.H. AiiA, an enzyme that inactivates the acylhomoserine lactone quorum-sensing signal and attenuates the virulence of Erwinia carotovora. Proc. Natl. Acad. Sci. USA 2000, 97, 3526-3531. [CrossRef]

10. Dong, Y.H.; Wang, L.H.; Xu, J.L.; Zhang, H.B.; Zhang, X.F.; Zhang, L.H. Quenching quorum-sensing-dependent bacterial infection by an $N$-acyl homoserine lactonase. Nature 2001, 411, 813-817. [CrossRef]

11. Fetzner, S. Quorum quenching enzymes. J. Bacteriol. 2015, 201, 2-14. [CrossRef] [PubMed]

12. Dong, Y.H.; Zhang, L.H. Quorum sensing and quorum-quenching enzymes. J. Microbiol. 2005, 43, $101-109$. [PubMed]

13. Cai, X.; Yu, M.; Shan, H.; Tian, X.; Zheng, Y.; Xue, C.; Zhang, X. Characterization of a Novel N-Acylhomoserine Lactonase RmmL from Ruegeria mobilis YJ3. Mar. Drugs 2018, 16, 370. [CrossRef] [PubMed]

14. Huang, W.; Lin, Y.; Yi, S.; Liu, P.; Shen, J.; Shao, Z.; Liu, Z. QsdH, a novel AHL lactonase in the RND-type inner membrane of marine Pseudoalteromonas byunsanensis strain 1A01261. PLoS ONE 2012, 7, e46587. [CrossRef] [PubMed]

15. De la Haba, R.R.; Sanchez-Porro, C.; Marquez, M.C.; Ventosa, A. Taxonomic study of the genus Salinicola: transfer of Halomonas salaria and Chromohalobacter salarius to the genus Salinicola as Salinicola salarius comb. nov. and Salinicola halophilus nom. nov., respectively. Int. J. Syst. Evol. Microbiol. 2010, 60, 963-971. [CrossRef] [PubMed]

16. Zhang, H.B.; Wang, L.H.; Zhang, L.H. Genetic control of quorum-sensing signal turnover in Agrobacterium tumefaciens. Proc. Natl. Acad. Sci. USA 2002, 99, 4638-4643. [CrossRef] [PubMed]

17. Joseph, T.C.; Baby, A.; Reghunathan, D.; Varghese, A.M.; Murugadas, V.; Lalitha, K.V. Draft Genome Sequence of the Halophilic and Highly Halotolerant Gammaproteobacteria Strain MFB021. Genome Announc. 2014, 2. [CrossRef] [PubMed]

18. Anan'ina, L.N.; Plotnikova, E.G.; Gavrish, E.; Demakov, V.A.; Evtushenko, L.I. Salinicola socius gen. nov., sp. nov., a moderately halophilic bacterium from a naphthalene-utilizing microbial association. Mikrobiologiia 2007, 76, 369-376. [CrossRef] [PubMed]

19. Sanchez-Porro, C.; De la Haba, R.R.; Soto-Ramirez, N.; Marquez, M.C.; Montalvo-Rodriguez, R.; Ventosa, A. Description of Kushneria aurantia gen. nov., sp. nov., a novel member of the family Halomonadaceae, and a proposal for reclassification of Halomonas marisflavi as Kushneria marisflavi comb. nov., of Halomonas indalinina as Kushneria indalinina comb. nov. and of Halomonas avicenniae as Kushneria avicenniae comb. nov. Int. J. Syst. Evol. Microbiol. 2009, 59, 397-405. [PubMed]

20. Wang, W.Z.; Morohoshi, T.; Someya, N.; Ikeda, T. AidC, a novel N-acylhomoserine lactonase from the potato root-associated cytophaga-flavobacteria-bacteroides (CFB) group bacterium Chryseobacterium sp. strain StRB126. Appl. Environ. Microbiol. 2012, 78, 7985-7992. [CrossRef] [PubMed]

21. Park, S.Y.; Lee, S.J.; Oh, T.K.; Oh, J.W.; Koo, B.T.; Yum, D.Y.; Lee, J.K. AhlD, an N-acylhomoserine lactonase in Arthrobacter sp., and predicted homologues in other bacteria. Microbiology 2003, 149, 1541-1550. [CrossRef] [PubMed]

22. Kim, M.H.; Choi, W.C.; Kang, H.O.; Lee, J.S.; Kang, B.S.; Kim, K.J.; Derewenda, Z.S.; Oh, T.K.; Lee, C.H.; Lee, J.K. The molecular structure and catalytic mechanism of a quorum-quenching $N$-acyl-L-homoserine lactone hydrolase. Proc. Natl. Acad. Sci. USA 2005, 102, 17606-17611. [CrossRef] [PubMed]

23. Momb, J.; Wang, C.; Liu, D.; Thomas, P.W.; Petsko, G.A.; Guo, H.; Ringe, D.; Fast, W. Mechanism of the quorum-quenching lactonase (AiiA) from Bacillus thuringiensis. 2. Substrate modeling and active site mutations. Biochemistry 2008, 47, 7715-7725. [CrossRef] [PubMed]

24. Yang, J.; Yan, R.; Roy, A.; Xu, D.; Poisson, J.; Zhang, Y. The I-TASSER Suite: protein structure and function prediction. Nat. Methods 2015, 12, 7-8. [CrossRef] [PubMed] 
25. Palzkill, T. Metallo-beta-lactamase structure and function. Ann. NY Acad. Sci. 2013, 1277, 91-104. [CrossRef] [PubMed]

26. Zhang, J.; Wang, J.; Feng, T.; Du, R.; Tian, X.; Wang, Y.; Zhang, X. Heterologous Expression of the Marine-Derived Quorum Quenching Enzyme MomL Can Expand the Antibacterial Spectrum of Bacillus brevis. Mar. Drugs 2019, 17, 128. [CrossRef]

27. Jones, S.; Yu, B.; Bainton, N.J.; Birdsall, M.; Bycroft, B.W.; Chhabra, S.R.; Cox, A.J.R.; Golby, P.; Reeves, P.J.; Stephens, S.; et al. The lux autoinducer regulates the production of exoenzyme virulence determinants in Erwinia carotovora and Pseudomonas aeruginosa. EMBO J. 1993, 12, 2477-2482. [CrossRef]

28. Yin, J.; Chen, J.C.; Wu, Q.; Chen, G.Q. Halophiles, coming stars for industrial biotechnology. Biotechnol. Adv. 2015, 33, 1433-1442. [CrossRef]

29. Fujimoto, K.; Morita, T. Aerobic removal of technetium by a marine Halomonas strain. Appl. Environ. Microbiol. 2006, 72, 7922-7924. [CrossRef]

30. Tahrioui, A.; Schwab, M.; Quesada, E.; Llamas, I. Quorum sensing in some representative species of halomonadaceae. Life 2013, 3, 260-275. [CrossRef]

31. Llamas, I.; Quesada, E.; Martinez-Canovas, M.J.; Gronquist, M.; Eberhard, A.; Gonzalez, J.E. Quorum sensing in halophilic bacteria: detection of $N$-acyl-homoserine lactones in the exopolysaccharide-producing species of Halomonas. Extremophiles 2005, 9, 333-341. [CrossRef] [PubMed]

32. Fomenko, D.E.; Gladyshev, V.N. Identity and functions of CxxC-derived motifs. Biochemistry 2003, 42, 11214-11225. [CrossRef] [PubMed]

33. Wang, L.H.; Weng, L.X.; Dong, Y.H.; Zhang, L.H. Specificity and enzyme kinetics of the quorum-quenching $\mathrm{N}$-Acyl homoserine lactone lactonase (AHL-lactonase). J. Biol. Chem. 2004, 279, 13645-13651. [CrossRef] [PubMed]

34. Seo, M.; Lee, B.S.; Pyun, Y.; Park, H. Isolation and Characterization of N-Acylhomoserine Lactonase from the Thermophilic Bacterium, Geobacillus caldoxylosilyticus YS-8. Biosci. Biotechnol. Biochem. 2011, 75, 1789-1795. [CrossRef] [PubMed]

35. Stevens, D.A.; Hamilton, J.R.; Johnson, N.; Kim, K.K.; Lee, J.S. Halomonas, a newly recognized human pathogen causing infections and contamination in a dialysis center: Three new species. Medicine 2009, 88, 244-249. [CrossRef] [PubMed]

36. Wang, W.Z.; Morohoshi, T.; Ikenoya, M.; Someya, N.; Ikeda, T. AiiM, a novel class of N-acylhomoserine lactonase from the leaf-associated bacterium Microbacterium testaceum. Appl. Environ. Microbiol. 2010, 76, 2524-2530. [CrossRef]

37. Xavier, R.; Patrice, G. Deciphering key features in protein structures with the new ENDscript server. Nucleic Acids Res. 2014, 42, 320-324.

(C) 2019 by the authors. Licensee MDPI, Basel, Switzerland. This article is an open access article distributed under the terms and conditions of the Creative Commons Attribution (CC BY) license (http://creativecommons.org/licenses/by/4.0/). 